\title{
CONFIABILIDAD SÍSMICA DE PLATAFORMAS MARINAS CON DAÑO POR FATIGA PARA LA ELABORACIÓN DE PLANES DE INSPECCIÓN BASADOS EN RIESGO
}

\author{
Francisco L Silva González ${ }^{(1)}$ y Ernesto Heredia Zavoni ${ }^{(1)}$
}

\begin{abstract}
RESUMEN
En este trabajo se propone un método para estimar la probabilidad de falla global de plataformas marinas con daño acumulado por fatiga sometidas a cargas sísmicas. Se discute la variación de la confiabilidad estructural de una plataforma marina con el tiempo y se presenta el modelo para la evaluación de la confiabilidad de las juntas sometidas a daño acumulado por fatiga. Se propone una función de estado límite expresada en términos del cortante basal resistente del jacket y el actuante debido a excitaciones sísmicas. La probabilidad anual de falla de la plataforma, condicional a un estado de daño por fatiga en las juntas, se estima mediante simulación de Monte Carlo. Se utiliza un modelo de riesgo, expresado en términos de costos esperados de inspección, reparación y falla, para la selección de planes óptimos de inspección estructural. Se ilustra su aplicación en el diseño de planes de inspección para extender la vida de servicio de plataformas marinas.
\end{abstract}

\begin{abstract}
A limit state function is proposed for estimating the probability of failure of steel jacket platforms under seismic loading and subjected to fatigue damage. It is associated with the base shear capacity of the jacket. The evolution of platform reliability over time is discussed and the model to assess the probability of failure of joints due to fatigue damage is presented. The probability of failure of the platform, given a state of fatigue damage in the joints, is estimated by means of Monte Carlo simulations. A risk model, expressed in terms of expected inspection, repair and future costs, is used for selecting optimal inspection plans. An application to risk based inspection planning is given for extension of the service life of marine platforms.
\end{abstract}

\section{INTRODUCCIÓN}

Las plataformas marinas se encuentran sometidas a la acción cotidiana del oleaje, el cual genera un número muy grande de ciclos de esfuerzos de carga y descarga que se traducen en daño por fatiga. Este daño se manifiesta en la aparición y propagación de grietas que debilitan la capacidad de carga de los elementos estructurales. En el caso de las plataformas marinas de acero las grietas se forman en las juntas del jacket y los elementos tubulares conectados a dichas juntas pierden capacidad de carga. El efecto del daño por fatiga es la reducción de la capacidad resistente

Artículo recibido el 20 de septiembre de 2006 y aprobado para su publicación el 28 de agosto de 2007. Se aceptarán comentarios y/o discusiones hasta cinco meses después de su publicación.

${ }^{(1)}$ Programa de Exploración de Campos en Aguas Profundas. Dirección de Investigación y Posgrado. Instituto Mexicano del Petróleo. Eje central Lázaro Cárdenas 152, México DF, 07730, México. Email: flsilva@imp.mx, eheredia@imp.mx 
global de la plataforma y por lo tanto el aumento de su riesgo de falla al verse sometida a las demandas de carga producto de un sismo de gran magnitud.

Los objetivos de llevar a cabo inspecciones programadas de las juntas del jacket durante la vida de servicio de las plataformas marinas son detectar la presencia del daño, evaluar su impacto en la integridad estructural, y tomar decisiones respecto de acciones de mantenimiento o reparación a fin de garantizar los requerimientos de seguridad. Por tal razón, se requieren diseñar los planes de inspección para identificar las juntas que deben inspeccionarse y las fechas de inspección. Una herramienta que se ha utilizado en la elaboración de planes de inspección para daño por fatiga ante el peligro de huracanes y tormentas extratropicales es el análisis de riesgo (Skjong, 1985; Madsen et al, 1989; Faber et al, 2000). El análisis de riesgo permite obtener soluciones que optimizan la asignación de recursos económicos a los trabajos de inspección con el objetivo final de mantener niveles adecuados de seguridad estructural. La inspección de daños por fatiga, con base en criterios de análisis de riesgo, ha sido aplicada también en los casos en los que se requiere extender la vida de servicio de plataformas expuestas a peligros oceanográficos (Heredia et al, 2006). Se pueden elaborar planes de inspección para aquellas juntas del jacket que tienen una vida esperada por fatiga que no satisface los requisitos de vida de servicio adicional. De esta manera, con un monitoreo de la evolución del posible daño en dichas juntas, se puede mantener un nivel adecuado de seguridad estructural de la plataforma durante el tiempo adicional que se pretende continúe en operación.

Además de la fatiga, las plataformas marinas se encuentran sometidas a otros procesos de deterioro y acumulación de daño, entre los que se encuentran la corrosión, las abolladuras y pandeos por impacto de embarcaciones o caída de objetos, la socavación, etc. Recientemente se ha propuesto un método usando Redes Bayesianas para evaluar el efecto combinado de diferentes tipos de daño en la variación con el tiempo de la confiabilidad estructural de plataformas marinas ante peligros oceanográficos. Este método ha sido aplicado a la planeación de inspección basada en criterios de confiabilidad aceptable, pero aún no se han incorporado a un análisis de riesgo.

Los desarrollos alcanzados en materia de inspección basada en riesgo han sido enfocados solamente al caso de peligros oceanográficos. En este trabajo se aborda la formulación de planes de inspección basada en riesgo para plataformas marinas con daño acumulado por fatiga en zonas de peligro sísmico. Se plantea primero el método para estimar la variación de la confiabilidad estructural de una plataforma marina con el tiempo y se presenta el modelo para la evaluación de la confiabilidad de las juntas sometidas a daño acumulado por fatiga. Se propone luego una función de estado límite para calcular mediante simulación de Monte Carlo la probabilidad anual de falla de la plataforma, condicional a un estado de daño por fatiga en las juntas. Después se describe un modelo de riesgo basado en costos totales esperados para el diseño de un plan de inspección óptimo basado en costos esperados mínimos y se muestra su aplicación. Finalmente se presentan algunos comentarios y conclusiones.

\section{EVOLUCIÓN EN EL TIEMPO DE LA CONFIABILIDAD ESTRUCTURAL}

En el análisis de confiabilidad estructural es de relevancia tomar en cuenta que con el transcurso del tiempo las estructuras se deterioran progresivamente. Entre los principales tipos de daño acumulable se encuentra el debido a fatiga, el cual se produce principalmente por cargas hidrodinámicas de oleaje operacional. El daño por fatiga se inicia cuando aparecen grietas en las juntas de la estructura y el proceso de acumulación de daño continúa durante la vida de la plataforma con la formación de nuevas grietas y propagación de grietas existentes. La acumulación de daño produce un deterioro de la capacidad estructural de la plataforma que incrementa el riesgo de una falla durante eventos extremos como los sismos. 
Sea $p_{a}(j)$ la probabilidad anual de falla de la plataforma en el año $j$, definida como la probabilidad de que la carga lateral producto de la acción de un sismo exceda la capacidad resistente del jacket en un año determinado. Suponiendo independencia estadística entre eventos sísmicos máximos anuales, la probabilidad acumulada de falla en un periodo de $t$ años se puede estimar como:

$$
p_{f}(t)=1-\prod_{j=1}^{t}\left[1-p_{a}(j)\right]
$$

Sea $N_{d}$ el número de estados de daño por fatiga considerados en el análisis. Desde un punto de vista práctico, cada estado de daño se define por la falla por fatiga de una junta en particular o por la falla de un grupo de juntas. La falla por fatiga de una junta se define generalmente como el evento en el que la profundidad de la grieta por fatiga alcanza el espesor de los elementos tubulares de acero. Considerando que los estados de daño son mutuamente excluyentes, la probabilidad anual de falla en el año $t$ se puede expresar como sigue:

$$
p_{a}(t)=p_{a, 0} p_{n d}(t)+\sum_{i=1}^{N_{d}} p_{a, i} p_{d, i}(t)
$$

donde $p_{a, 0}$ es la probabilidad anual de falla dado que no existe daño por fatiga en la estructura; $p_{a, i}$ es la probabilidad anual de falla dado que existe el estado de daño por fatiga $i ; p_{d, i}(t)$ es la probabilidad de que el estado de daño por fatiga $i$ se presente en el año $t ; p_{n d}(t)$ es la probabilidad de que la estructura no tenga daño por fatiga en el año $t$,

$$
p_{n d}(t)=\prod_{i=1}^{N_{d}}\left(1-p_{d, i}(t)\right)
$$

Al suponer que los estados de daño son mutuamente excluyentes, la ecuación (2) no considera el efecto de la correlación entre dichos estados de daño, lo cual es una aproximación conservadora. Como se discutirá a continuación, la probabilidad de falla por fatiga en una junta dada puede estimarse mediante técnicas de confiabilidad usando modelos de mecánica de fractura (Kirkemo, 1988; Madsen et al, 1986; Heredia y Montes, 2004; Silva y Heredia, 2004). Nótese que el cálculo de la probabilidad anual de falla en la ecuación (2) requiere también estimar la probabilidad condicional de falla de la plataforma dado que se encuentra en un cierto estado de daño. En este trabajo, dicha probabilidad de falla se calcula a través de simulaciones de Monte Carlo.

\section{PROBABILIDAD DE FALLA POR FATIGA DE JUNTAS TUBULARES}

El criterio de falla por fatiga de una junta tubular adoptado en este trabajo es:

$$
a_{c}-a(t) \leq 0
$$

donde $a_{c}$ es la profundidad crítica de la grieta, considerada como el espesor del elemento tubular. En la ecuación (4), $a(t)$ es la profundidad de la grieta en el instante $t$, la cual se calcula de acuerdo con 
la ley de Paris-Erdogan (1963), basada en la teoría de la mecánica de la fractura elástica. La tasa de crecimiento del tamaño de grieta puede evaluarse como sigue:

$\frac{d a}{d N}=C(\Delta S Y(a, x) \sqrt{\pi a})^{n}$

donde $a$ es la profundidad de la grieta, $C$ y $m$ son parámetros que dependen del material, $N$ es el número de ciclos de esfuerzos, $\Delta S$ es el intervalo de esfuerzos de amplitud constante, y $Y(a, x)$ se conoce como factor de corrección geométrico finito el cual depende no sólo de la profundidad de grieta, $a$, sino también de un conjunto de variables denotadas por $x$ relacionadas con la geometría de la grieta y de la junta así como del intervalo de esfuerzos. Si el intervalo de esfuerzos es aleatorio y se considera que tiene una distribución de probabilidad de Weibull con factor de forma $B$ y factor de escala $D$, se puede demostrar que la ecuación (5) se transforma en (Kirkemo, 1988; Silva y Heredia, 2004):

$$
M_{f}(X, t)=\int_{a_{0}}^{a_{c}} \frac{d a}{Y^{m}(\sqrt{\pi a})^{m}}-C D^{m} \Gamma\left(\frac{m}{B}+1\right) \nu_{0} t
$$

donde $a_{0}$ es el tamaño de la grieta inicial en el instante $t=0, \Gamma(\cdot)$ es la función gamma y $v_{0}$ es la tasa media de cruces por cero de los esfuerzos. $\bar{X}$ denota el vector de variables aleatorias consideradas en el problema, en general: $\bar{X}=\left(a_{0}, a_{c}, Y, B, D, m, C, v_{0}\right)$. La expresión 6 define el margen de seguridad por fatiga. Así, la probabilidad de falla por fatiga, es decir, la probabilidad de que la profundidad de grieta exceda al tamaño crítico en el instante $t$ es:

$P\left(a_{c}-a(t) \leq 0\right)=P\left(M_{f}(t) \leq 0\right)=p_{d}(t)$ manera:

La probabilidad de falla está relacionada con el índice de confiabilidad de la siguiente

$$
\beta(t)=-\Phi^{-1}\left(p_{d}(t)\right)
$$

En este trabajo, el cálculo de la probabilidad de falla por fatiga (ecuación 7) se lleva a cabo con el método SORM (Second Order Reliability Method) (Madsen et al, 1986) y se considera que el vector de variables aleatorias es: $\bar{X}=\left(a_{0}, B, D, m, C\right)$. Para aplicar el método SORM es necesario conocer las distribuciones de probabilidad de cada variable; las distribuciones generalmente usadas se encuentran en la literatura, ver por ejemplo Kirkemo (1988) y Silva y Heredia (2004).

\section{PROBABILIDAD CONDICIONAL DE FALLA DE LA PLATAFORMA}

La probabilidad anual de falla por sismo dado que la plataforma se encuentra en algún estado de daño por fatiga, o dado que no existe daño, se puede evaluar mediante simulación de Monte Carlo como sigue

$$
p_{a, i}=P[g(Z) \leq 0] \quad ; \quad i=0,1, \ldots, N_{d}
$$


donde $g(Z)$ es una función de estado límite asociada a una falla del jacket. La función de estado límite se define en términos del cortante basal resistente del jacket y del cortante basal actuante producto de un sismo cuya intensidad es la máxima esperada en un año. Se propone la siguiente función:

$g(\mathbf{Z})=B_{R} \frac{R}{F_{e}}-B_{S} S$

donde $Z$ es un vector de variables aleatorias, e.g. $\mathbf{Z}=\left(R, S, B_{R}, B_{S}\right), R$ es la capacidad nominal del jacket en condición intacta o dañada, medida en términos del cortante basal resistente, $S$ es la carga nominal que induce un sismo sobre el jacket expresada en términos del cortante basal actuante, y $F_{e}$ es un factor de capacidad estructural que considera los efectos de ductilidad y resistencia residual, $F_{e} \leq 1$ (Bea, 1997). La incertidumbre en los modelos analíticos con los que se evalúan $R$ y $S$ se introduce en la ecuación (10) mediante los sesgos en la resistencia del jacket, $B_{R}$, y en la carga sísmica actuante, $B_{S}$. El sesgo se define como la relación entre una variable aleatoria y el valor nominal, considerado éste como el valor predicho por un modelo analítico

Cabe mencionar que la caracterización del cortante basal resistente debe tomar en cuenta la capacidad de deformación dúctil del sistema estructural. En el caso de plataformas marinas, el criterio de comparar cortante basal actuante y resistente es congruente con el estado del arte en las prácticas de ingeniería de la industria. A fin de considerar el efecto de la ductilidad y el comportamiento no-lineal del sistema en la caracterización del cortante resistente, se introduce el factor de capacidad $F_{e}$ (Bea, 1997). Este factor incorpora las características de ductilidad y resistencia residual de la plataforma cuando ésta se sujeta a cargas sísmicas transitorias. Este factor modifica la capacidad del jacket derivada de un análisis pushover para tomar en cuenta principalmente el comportamiento histerético no lineal característico de una plataforma. El factor $F_{e}$ se calcula como:

$F_{e}=(\mu \alpha)^{-1}$

donde $\mu$ es la ductilidad global y $\alpha$, generalmente menor que 1.0 , es la relación de resistencia residual de la plataforma, definida como el cociente del área debajo de la curva de resistencia ultima, $A$, y el área bajo una curva elastoplástica con el mismo intervalo elástico y el mismo desplazamiento lateral máximo, $A_{e p}$ (ver figura 1). En este trabajo, estos parámetros se modelan como variables aleatorias a fin de considerar las incertidumbres en su estimación.

A continuación se describe la manera en que se modelan cada una de las variables aleatorias que intervienen en la función de estado límite (ecuación 10). El cortante basal resistente $R$ se debe caracterizar tanto para los estados de daño a considerar en el análisis como para el estado intacto o sin daño de la plataforma; esto con el fin de poder estimar las probabilidades condicionales de falla dado un cierto estado de daño o dada la condición de plataforma sin daño. La resistencia global de la plataforma, $R$, se considera una variable aleatoria lognormal. El valor medio de $R$ se toma igual a la resistencia determinada con un análisis inelástico no lineal de empuje lateral conocido también como análisis pushover. Para el caso de la estructura dañada se considera que los elementos asociados a las juntas dañadas por fatiga no tienen capacidad de transmisión de carga y por lo tanto se retiran del modelo estructural. Cuando se analiza el efecto del daño por fatiga en un elemento tubular conectado a una junta crítica del jacket de una plataforma, es común suponer para el análisis de resistencia última que el elemento ha perdido completamente la capacidad de transmisión de carga; lo que es equivalente a retirarlo del modelo estructural para el cálculo de la capacidad 
resistente. Esta condición corresponde al caso de un daño total. Debido a la redundancia de plataformas estructuradas adecuadamente, no hay diferencias significativas entre la estimación de la capacidad estructural al retirar completamente un elemento tubular y la consideración de estados parciales de daño en el elemento. En rigor, la probabilidad de falla que se evalúa de esta manera, es mayor que la que corresponde a los estados de daño parciales.

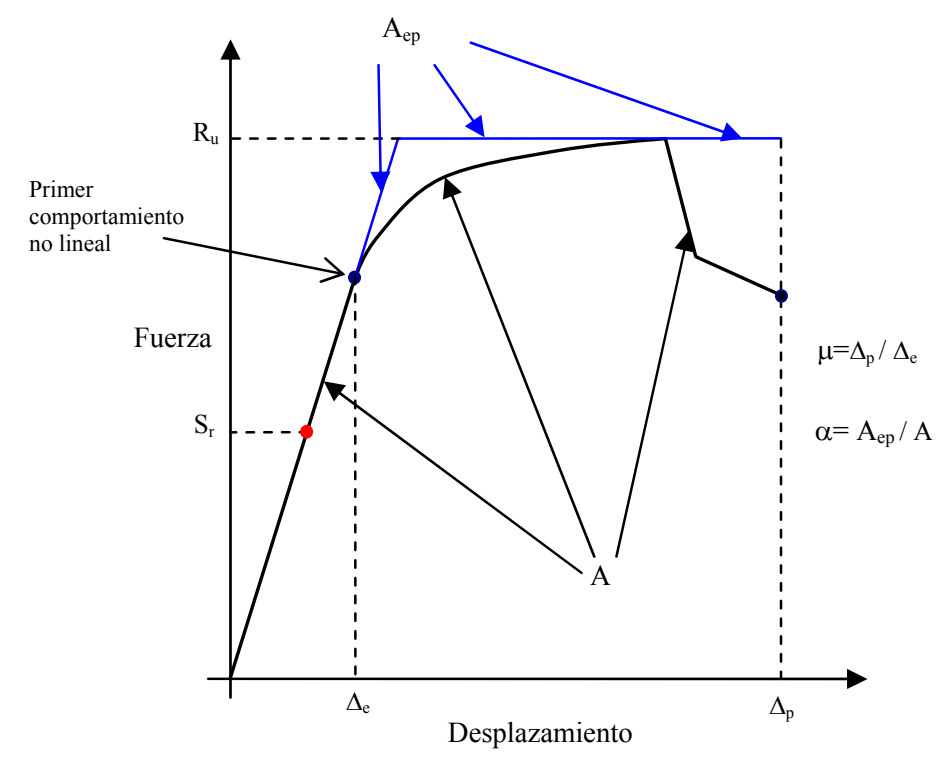

Figura 1. Curva carga vs. desplazamiento lateral de la plataforma.

La distribución de probabilidad de las fuerzas que inducen los eventos sísmicos de intensidad máxima anual sobre la plataforma, $S$, es modelada razonablemente por una distribución lognormal (Bea, 1992). La media de esta variable se calcula en este trabajo a través de la mediana de la carga sísmica actuante implícita en la formulación de Bea (1992) para la Sonda de Campeche

$m_{S}=S_{r} \operatorname{Exp}\left[-z_{D} \sigma_{L n S}\right]$

donde $S_{r}$ es el cortante basal de referencia obtenido con un espectro de diseño y $z_{D}$ es un factor que depende del periodo de retorno usado para definir dicho espectro; por ejemplo, para un periodo de retorno $T_{R}=200$ años, $z_{D}=2.57$. El coeficiente de variación del logaritmo de la carga sísmica se determina con:

$\sigma_{L n S}^{2}=\sigma_{L n S E}^{2}+\sigma_{L n G S}^{2}+\sigma_{L n R S}^{2}$

donde $\sigma_{\text {LnSE }}^{2}$ es la incertidumbre en la aceleración pico en la roca basal debida a las características de la fuente sísmica y a la atenuación de la fuente al sitio, y $\sigma_{L n G S}^{2}$ es la incertidumbre de las ordenadas del espectro de respuesta debidas a la geología local y a las condiciones del suelo. En la ecuación (13), $\sigma_{\text {LnRS }}^{2}$ es la incertidumbre sobre las características de desempeño de la plataforma, que contribuyen a determinar las fuerzas inducidas, así como del método para calcular dichas fuerzas en los elementos que componen la plataforma. Esta incertidumbre incluye incertidumbres en la evaluación de modos, periodos y masas, combinación de modos, amortiguamiento (estructural, hidrodinámico, cimentación) y las incertidumbres en la reducción por ductilidad de las ordenadas del espectro elástico. 
La ductilidad $\mu$ se modeló como una variable aleatoria lognormal. La media de la ductilidad se estimó a través de un análisis de empuje lateral de la plataforma. El valor de la media de la ductilidad se tomó como el cociente del desplazamiento lateral máximo (medido a nivel de la cubierta) que puede desarrollarse sin colapso de la plataforma entre el desplazamiento en el que aparece el primer comportamiento no lineal. La relación de resistencia residual $\alpha$ se consideró una variable aleatoria con distribución lognormal truncada definida en $0 \leq \alpha \leq 1$. El valor que se obtiene de los resultados de un análisis de resistencia última se tomó aquí como valor medio de relación de resistencia residual.

\section{CASO DE ESTUDIO}

Considérese una plataforma marina instalada en el año 1980, en un tirante de agua de 25 m en la Sonda de Campeche. Es una plataforma de acero de ocho piernas, con dos marcos longitudinales, cuatro transversales, y dos cubiertas que se encuentran en elevaciones $+19.100 \mathrm{y}+23.999 \mathrm{~m}$. Las piernas de la plataforma son de 51.25 pulgadas $(130.18 \mathrm{~cm})$ de diámetro y espesor de 0.625 pulgadas $(1.59 \mathrm{~cm})$. La cimentación esta constituida por ocho pilotes: cuatro interiores y cuatro de esquina. Los pilotes de esquina son perfiles tubulares OC de 48 pulgadas $(121.92 \mathrm{~cm})$ de diámetro y espesores que varían entre 1.25 a 2.50 pulgadas $(3.18$ y $6.35 \mathrm{~cm}$, respectivamente) hincados a una profundidad de $93.15 \mathrm{~m}$ bajo el nivel del lecho marino. Los pilotes interiores están formados por perfiles tubulares OC del mismo diámetro (48 pulgadas), y se encuentran hincados a una profundidad de $73.23 \mathrm{~m}$ bajo el nivel del lecho marino; los espesores de estos pilotes varían de 1.25 $(3.18 \mathrm{~cm})$ a 2.50 pulgadas $(6.35 \mathrm{~cm})$. La configuración estructural se muestra en la figura 2 . El periodo de vibración asociado al primer modo en dirección transversal es $1.70 \mathrm{~s}$, del segundo modo es $1.43 \mathrm{~s}$ en dirección longitudinal y del tercer modo es $1.37 \mathrm{~s}$ en torsión.

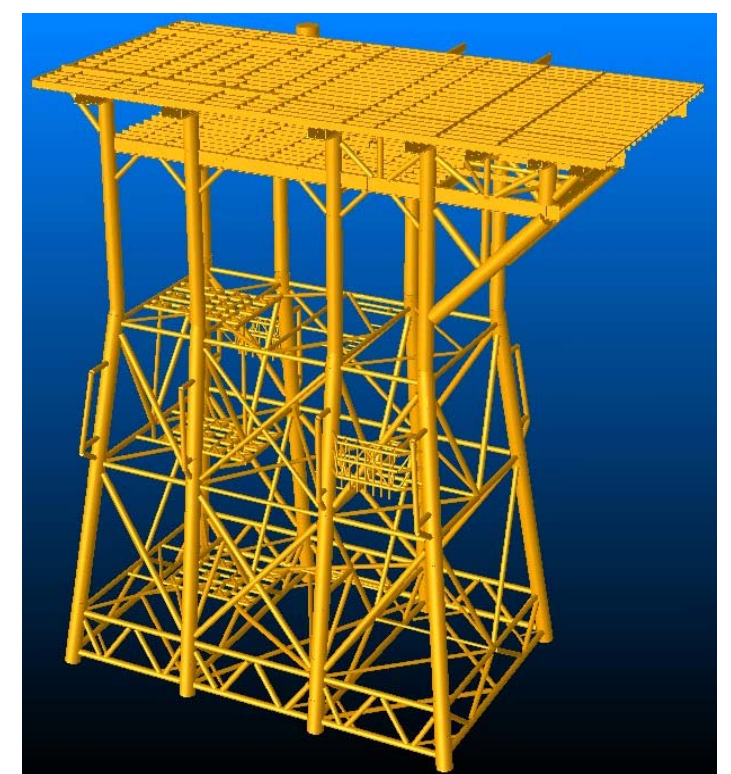

Figura 2. Modelo estructural de la plataforma en estudio

Se han realizado estudios para establecer las condiciones sísmicas que deben considerarse para diseñar y evaluar plataformas marinas en México. El peligro sísmico en la Bahía de Campeche está asociado a tres tipos de fuentes sísmicas (figura 3). El primer tipo de fuente corresponde a la 
zona de subducción en la costa oeste del Pacífico mexicano. Los sismos en esta zona ocurren a profundidades de $15 \mathrm{~km}$ a $20 \mathrm{~km}$ y con magnitudes que pueden ser del orden de hasta $\mathrm{M}=8.2$. La segunda fuente está asociada con la placa litosférica donde los sismos se presentan a profundidades entre $60 \mathrm{~km}$ y $250 \mathrm{~km}$ con magnitudes cercanas a M=7.5. La tercera fuente es el cinturón volcánico transcontinental mexicano, donde los sismos se originan en profundidades del orden de $20 \mathrm{~km}$ con magnitudes mayores que $\mathrm{M}=6.7$ (Chávez, 1997).

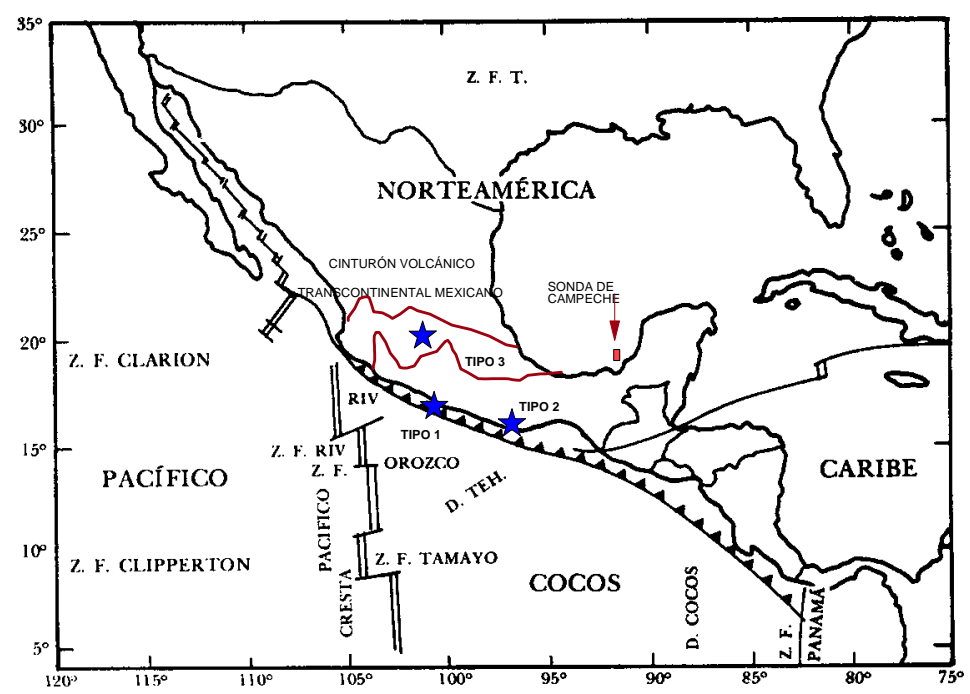

Figura 3. Zonas que contribuyen al peligro sísmico de la Sonda de Campeche.

Supóngase que se ha cumplido la vida de servicio de la plataforma y se requiere continuar con su operación por un tiempo adicional de 20 años. Se utilizaron los resultados de un análisis espectral para calcular la vida esperada por fatiga de las juntas críticas e identificar las que requieren de un plan de inspección. Estas juntas son aquellas que tienen una vida esperada por fatiga menor que la requerida de acuerdo con la vida remanente de 20 años de la plataforma. Un criterio para identificar dichas juntas es seleccionar aquellas cuya vida esperada por fatiga es menor que la vida total de servicio de la plataforma, entendida esta última como la suma de la edad de la plataforma más el tiempo adicional que se planea que la instalación continúe en operación. Un criterio más restrictivo considera el re-uso de estructuras (API RP-2A, 1993). En tal caso se exige que la vida esperada por fatiga de las juntas sea mayor que dos veces la edad de la plataforma más dos veces su vida adicional. Este criterio se usó en este trabajo para seleccionar las juntas. Para una vida adicional de 20 años, se seleccionaron entonces aquellas juntas con vida esperada por fatiga menor que 92 años. En la figura 4 se muestran las tres juntas identificadas así como los elementos tubulares del jacket que han sido removidos para modelar los estados de daño asociados con la falla de dichas juntas. Se consideraron tres estados de daño: uno asociado a la falla por fatiga de cada una de estas tres juntas.

El análisis de empuje lateral se llevó a cabo con un perfil de carga obtenido de un análisis dinámico espectral utilizando el espectro de la norma NRF-003-PEMEX-2000 (PEMEX, 2000), cuyo periodo de retorno es $T_{R}=200$ años. En la figura 5 se presenta la curva de resistencia última obtenida del análisis de empuje lateral de la estructura sin daño, en la dirección más crítica: la longitudinal. En cada paso de este análisis, el perfil de carga se multiplicó por un factor de carga y se determinó el desplazamiento de la cubierta superior. El análisis se efectuó considerando la formación de rótulas plásticas en los elementos tubulares del jacket hasta que la plataforma se convierte en un mecanismo inestable. La deformación que corresponde a este estado es la 
deformación última con la que se estimó la ductilidad. En la figura 5 se indica el punto de la curva que corresponde al primer comportamiento no lineal de la plataforma. Se muestra también la curva que correspondería a un comportamiento elastoplástico ideal de la plataforma, con el mismo intervalo elástico y el mismo desplazamiento lateral máximo obtenidos en el análisis de resistencia última.

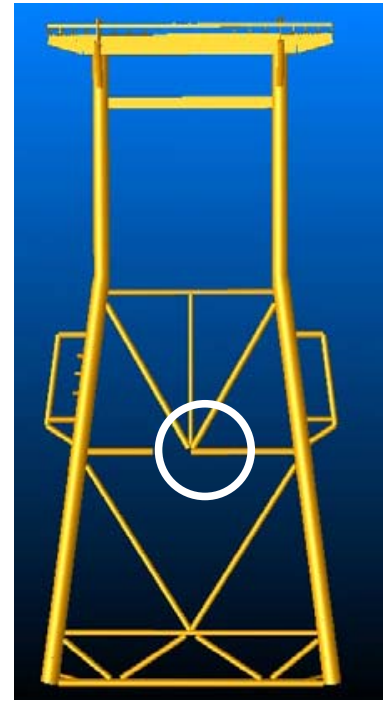

(a) Estado de daño 1: junta $\mathrm{N}^{\circ} 1$ dañada

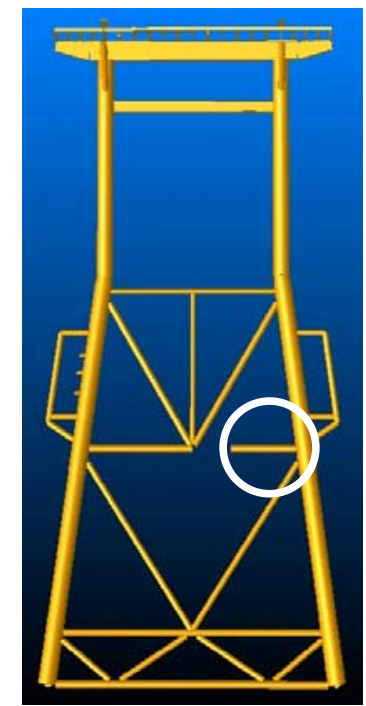

(b) Estado de daño 2: junta $\mathrm{N}^{\circ} 2$ dañada

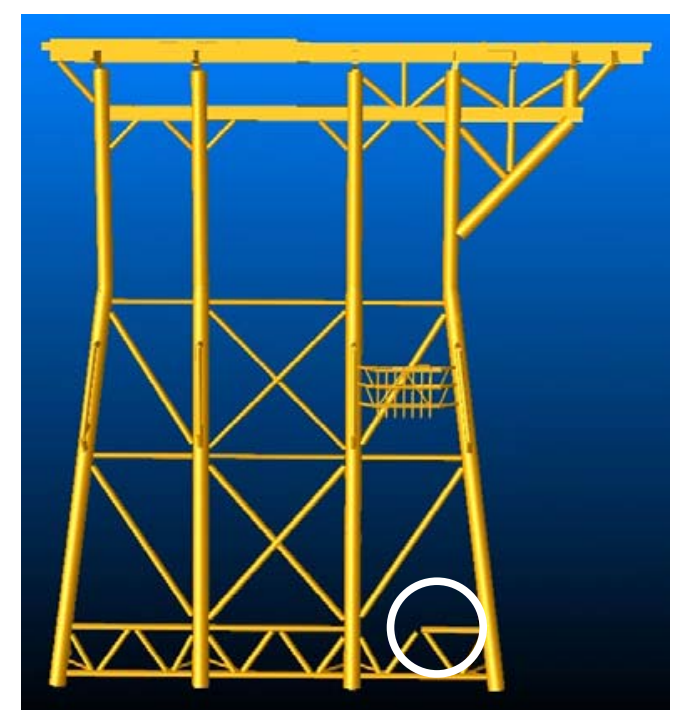

(c) Estado de daño 3: junta $\mathrm{N}^{\circ} 3$ dañada

Figura 4. Elementos removidos para modelar los estados de daño

Los resultados de los análisis de resistencia última de la plataforma para cada estado de daño se resumen en la tabla 1 . Con base en estos resultados se caracterizó el valor esperado del cortante resistente último, así como los valores esperados de la ductilidad y de la relación de resistencia residual de la plataforma. Para la simulación de Monte Carlo, la incertidumbre del logaritmo de la resistencia se tomó igual a $\sigma_{L n R}=0.15$, valor asociado con el desempeño de diagonales de 
contraventeo vertical cargadas sísmicamente en compresión (Bea, 1997). La varianza $\sigma_{\text {LnRS }}^{2}$ en la ecuación (13), se estimó considerando que las incertidumbres en: 1) la evaluación de modos, periodos y masas; 2) la combinación de modos; 3 ) el amortiguamiento (estructural, hidrodinámico y cimentación); y 4) la reducción por ductilidad de las ordenadas del espectro elástico, están dadas cada una de ellas por una desviación estándar igual a 0.15 . Por lo tanto, $\sigma_{\text {LnRS }}=0.3$.

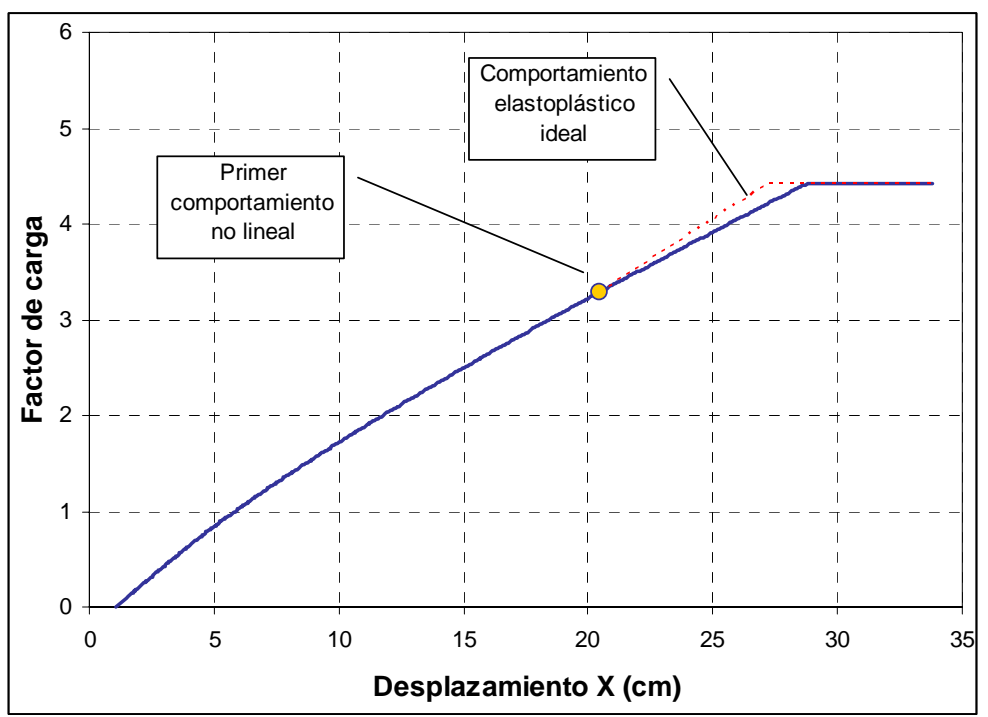

Figura 5. Curva de resistencia última de la estructura sin daño.

Tabla 1. Resultados del análisis de resistencia última (dirección longitudinal)

\begin{tabular}{lcccc}
\hline Condición & $\begin{array}{c}\text { Cortante de } \\
\text { referencia } S_{\mathrm{r}} \\
{[\mathrm{t}]}\end{array}$ & $\begin{array}{c}\text { Cortante de } \\
\text { resistencia } \\
\text { última [t] }\end{array}$ & $\mu$ & $\alpha$ \\
\hline Intacta & 431 & $1,909.33$ & 1.65 & 0.98 \\
Daño 1 & 431 & $1,900.71$ & 1.40 & 0.98 \\
Daño 2 & 431 & $1,900.71$ & 1.39 & 0.94 \\
Daño 3 & 431 & $1,887.78$ & 1.42 & 0.98 \\
\hline
\end{tabular}

Se consideró que $\sigma_{L n S E}=1.03$ asociada a la fuente sísmica 3 (Chávez, 1997) y $\sigma_{L n G S}=0.5$;

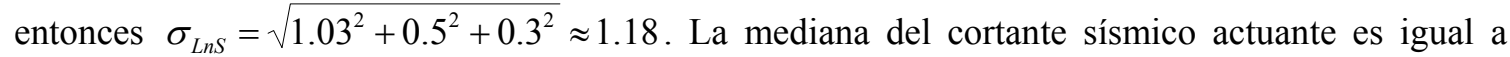
(ecuación 12): $m_{S}=431 \operatorname{Exp}[-2.57 \times 1.18]=20.77 t$. Los coeficientes de variación de $\mu \mathrm{y} \alpha$ se supusieron iguales a 0.1 . La mediana del sesgo de la carga actuante $B_{S}$ se tomó igual a 1.0 y su coeficiente de variación igual a 0.15 ; la mediana del sesgo de la resistencia $B_{R}$ se consideró igual a 1.4 y su coeficiente de variación igual a 0.15 (Bea, 1997).

El factor de amplificación dinámica se calculó con los valores de ductilidad y de relación de resistencia residual generados en cada simulación. Las probabilidades de falla calculadas con base en $2 \times 10^{6}$ simulaciones, y los correspondientes índices de confiabilidad, se muestran en la tabla 2 . Se observa el incremento en la probabilidad anual de falla condicional a los tres estados de daño, como resultado de una capacidad resistente del jacket menor. 
Tabla 2. Probabilidades condicionales de falla

\begin{tabular}{lcc}
\hline \multicolumn{1}{c}{ Condición } & $p_{a}$ & $\beta$ \\
\hline Intacta & $8.00 \times 10^{-6}$ & 4.31 \\
Daño 1 & $1.25 \times 10^{-5}$ & 4.21 \\
Daño 2 & $1.85 \times 10^{-5}$ & 4.12 \\
Daño 3 & $1.10 \times 10^{-5}$ & 4.24 \\
\hline
\end{tabular}

Con el fin de estudiar la importancia de la incertidumbre de la ductilidad y del factor de resistencia residual, se efectuó un análisis de sensibilidad de la probabilidad condicional de falla ante diferentes valores de los coeficientes de variación de $\mu$ y $\alpha$. La figura 6 muestra el cambio de la probabilidad anual de falla con los coeficientes de variación usados para la ductilidad y el factor de resistencia residual. Se observa que el efecto de la aleatoriedad en estos parámetros es incrementar la probabilidad anual de falla. Por ejemplo, para el caso del estado de daño $3, p_{a}=5.5 \times 10^{-6}$ si dichos parámetros se consideran deterministas, y $p_{a}=2 \times 10^{-5}$ para coeficientes de variación del $20 \%$. La probabilidad anual de falla puede variar en un orden de magnitud ante tal consideración en la incertidumbre de la ductilidad y el factor de resistencia residual. Una caracterización adecuada de estos parámetros es por lo tanto importante para la estimación de la probabilidad de falla de la plataforma. Resultados similares se muestran en la figura 7 en términos del índice de confiabilidad.

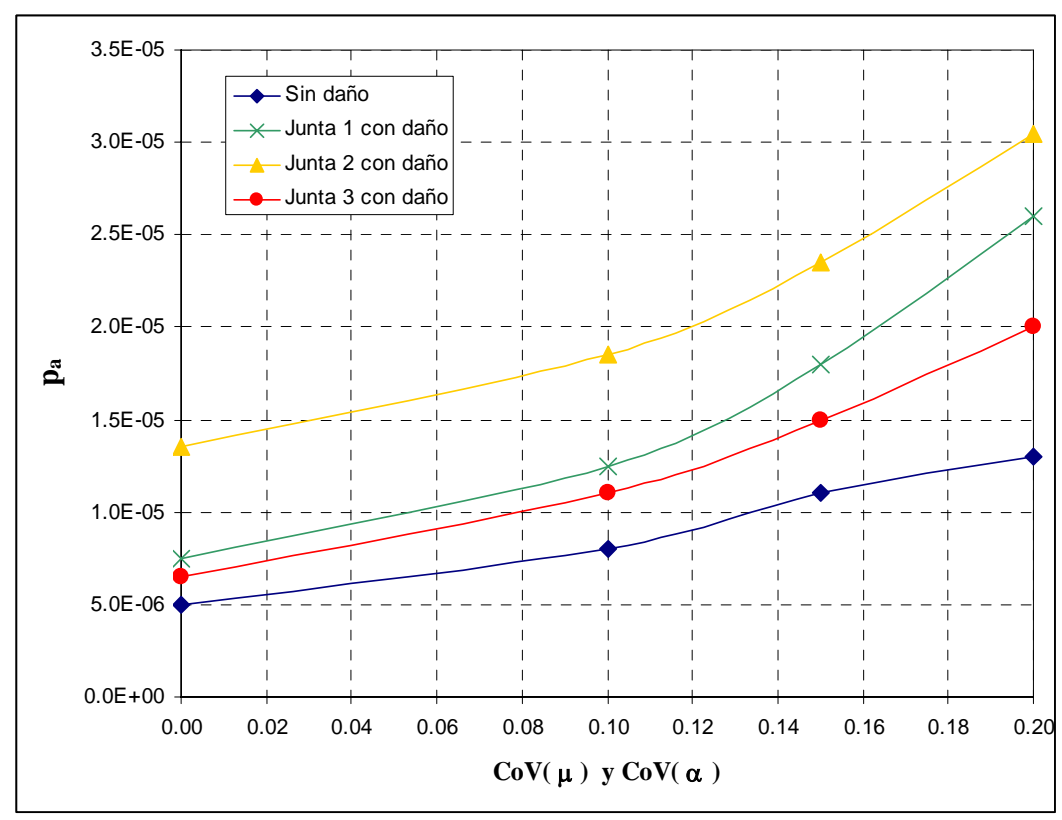

Figura 6. Variación de la probabilidad anual de falla con los coeficientes de variación de la ductilidad y del factor de resistencia residual 


\section{PLANES DE INSPECCIÓN BASADOS EN RIESGO}

Una vez que se han calculado las probabilidades condicionales de falla dados diferentes estados de daño y la probabilidad acumulada de falla en las ecuaciones (1) y (2), se puede hacer uso del análisis de riesgo para planear la inspección de las juntas. El análisis de riesgo permite seleccionar planes de inspección óptimos que satisfacen los requisitos de seguridad y que minimizan costos esperados. El riesgo se estima en términos del costo total esperado a lo largo de la vida de servicio de la plataforma, incluyendo los costos esperados de inspección, de reparación y de falla, como sigue:

$$
E\left[C_{T}\right]=E\left[C_{I}\right]+E\left[C_{R}\right]+E\left[C_{F}\right]
$$

donde $C_{T}, C_{I}, C_{R}$ y $C_{F}$ son el costo total, el de inspección, el de reparación y el costo futuro asociado con las pérdidas por una falla de la plataforma, respectivamente; $E[\cdot]$ es el operador valor esperado. El plan de inspección óptimo, que indica los años de inspección de las juntas, se selecciona de manera de minimizar el costo total esperado, sujeto a la condición de satisfacer requerimientos mínimos de confiabilidad a nivel de las juntas.

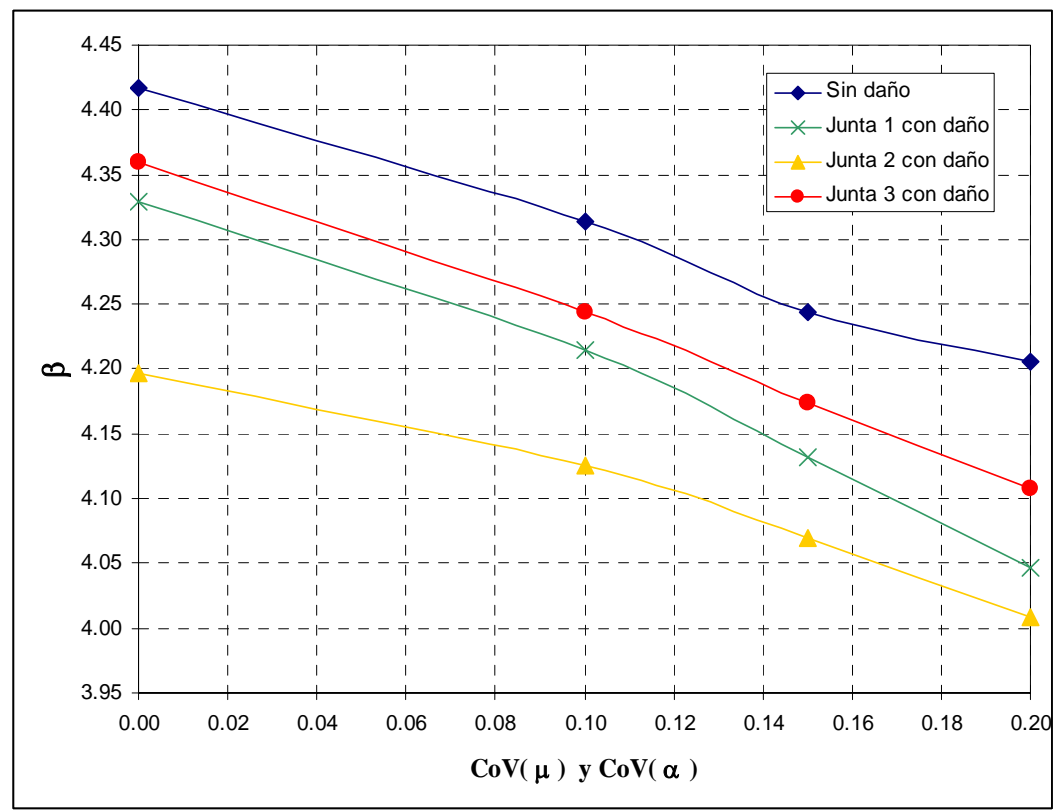

Figura 7. Variación del índice de confiabilidad con los coeficientes de variación de la ductilidad y del factor de resistencia residual

Supongamos que se elaboran planes de inspección de daño por fatiga para $M$ juntas. Sea $T_{i k}$; $i=1,2, \ldots, N_{k} ; k=1,2, \ldots, M$, el tiempo de inspección $i$ de la junta $k$ en el intervalo de interés $0 \leq t \leq L$ ( $L$ es la vida remanente de la plataforma), y sea $N_{k}$ el número de inspecciones de la junta $k$. El valor presente de los costos esperados de inspección se puede estimar como sigue (Enevoldsen y Sørensen, 1994):

$E\left[C_{I}\right]=\sum_{k=1}^{M} \sum_{i=1}^{N_{k}} C_{I, i k}(q)\left[1-p_{f}\left(T_{i k}\right)\right] \frac{1}{\left(1+r_{i k}\right)^{T_{i k}}}$ 
donde $C_{I, i k}(q)$ es el costo de inspección promedio por junta en el tiempo $T_{i k}$ asociado a una calidad de inspección $q, p_{f}\left(T_{i, k}\right)$ es la probabilidad acumulada de falla de la plataforma a nivel global en el tiempo $T_{i k}, \mathrm{y} r_{i k}$ es la tasa anual neta de descuento o interés en $T_{i k}$.

El valor presente de los costos esperados de reparación se calcula por medio de (Enevoldsen y Sørensen, 1994):

$$
E\left[C_{R}\right]=\sum_{k=1}^{M} \sum_{i=1}^{N_{k}} C_{R, i k} p_{R k}\left(T_{i k}\right)\left[1-p_{f}\left(T_{i k}\right)\right] \frac{1}{\left(1+r_{i k}\right)^{T_{i k}}}
$$

donde $C_{R, i k}$ es el costo de reparación de la junta $k$ en $T_{i k}$ y $p_{R k}\left(T_{i k}\right)$ es la probabilidad de reparación de la junta $k$ en $T_{i k}$. Por lo tanto, obsérvese que el valor presente de los costos esperados de reparación y por lo tanto el plan de inspección óptimo depende de la filosofía y del tipo de reparación. En este estudio la probabilidad de reparación $p_{R k}\left(T_{i k}\right)$ se calcula en términos del factor de reserva de resistencia mínimo admisible de la plataforma $\left(R S R_{a d m}\right)$. El $R S R$ se define como:

$$
R S R=\frac{R_{U}}{S_{r}}
$$

donde $R_{U}$ es el cortante resistente último de la plataforma y $S_{r}$ es un cortante basal de referencia asociado a un periodo de retorno dado. Si $S_{r}$ es constante en el tiempo, la probabilidad de reparación de la junta $k$ en $T_{i k}$ es:

$$
p_{R, k}\left(T_{i k}\right)=P\left[R_{U}\left(T_{i k}\right) \leq R S R_{a d m} \times S_{r}\right]
$$

donde $R_{U}\left(T_{i k}\right)$ denota la resistencia lateral última de la plataforma en el tiempo $T_{i k}$. En la ecuación (18), la decisión de reparación depende solamente de si la resistencia en la base de la plataforma, dado un cierto estado de daño, satisface el $R S R_{a d m}$, lo cual es la práctica actual en la industria nacional para efectos de evaluación estructural. Se debe hacer notar que el costo de reparación depende de la magnitud del daño de la junta $k$ en el tiempo de inspección $T_{i k}$. Por otro lado, la probabilidad de que la junta se repare, $p_{R, k}\left(T_{i k}\right)$, depende de si la resistencia de la plataforma, dado el daño de cierta magnitud (por ejemplo el tamaño de la grieta) en la junta $k$, es suficiente para satisfacer el factor de reserva de resistencia admisible, $R S R_{a d m}$. En la práctica sólo existen dos acciones de reparación: el esmerilado de grietas pequeñas ó la colocación de una abrazadera cuando se considera que el daño es moderado o severo. En el procedimiento establecido en este trabajo, el cortante resistente se evalúa para estados de daño en los que los elementos se retiran completamente de la estructura, lo que corresponde a un daño total. Por ello, sólo se considera una acción de reparación que es la colocación de una abrazadera. La inclusión de estados intermedios de daño en el procedimiento, permitiría, mediante un proceso de optimización, establecer un tamaño de grieta óptimo para efectuar un esmerilado o colocar una abrazadera. Dicho problema está fuera del alcance de este trabajo.

La norma de referencia NRF-003-PEMEX-2000 (PEMEX, 2000) establece valores de $R S R_{\text {adm }}$ para plataformas existentes, los cuales están asociados a índices de confiabilidad obtenidos con base en criterios de minimización del riesgo. Para calcular $p_{R k}\left(T_{i k}\right)$ se supone que $R_{U}\left(T_{i k}\right)$ es una variable aleatoria lognormal con valor medio dado por: 


$$
E\left[R_{U}\left(T_{i k}\right)\right]=E\left[R_{U} \mid \text { no daño }\right] P\left[M_{f}\left(T_{i k}\right)>0\right]+E\left[R_{U} \mid M_{f}\left(T_{i k}\right) \leq 0\right] P\left[M_{f}\left(T_{i k}\right) \leq 0\right]
$$

Los valores medios condicionales de resistencia última pueden obtenerse del análisis de resistencia última de la plataforma considerando o no daño por fatiga. $M_{f}\left(T_{i k}\right)$ es el margen de falla asociado con la falla por fatiga de la junta $k$ en el tiempo $T_{i k}$.

En términos de probabilidades anuales de falla, $p_{a}(j)(j=1,2, \ldots, L)$, el valor presente de los costos esperados de falla están dados por (Enevoldsen y Sørensen, 1994):

$$
E\left[C_{F}\right]=\sum_{j=1}^{L} C_{F}(j) p_{a}(j) \frac{1}{\left(1+r_{j}\right)^{j}}
$$

donde $C_{F}(j)$ es el costo futuro asociado a la falla estructural en el año $j$, y $r_{j}$ es la tasa anual neta de descuento en el año $j$. Los costos de falla para cada año, $C_{F}(\mathrm{j})$, consideran en general las pérdidas económicas debido a la interrupción de la producción, costos por pérdida de vidas humanas o lesiones, daño al medio ambiente, y costos por daños a la instalación, pérdidas de equipo, materiales y pozos.

Las probabilidades de falla $p_{f}\left(T_{i, k}\right)$ y $p_{a}(j)$ de las ecuaciones (15), (16) y (20) deben considerar los resultados de las inspecciones efectuadas en el pasado así como todos los posibles resultados de las inspecciones futuras (Moan et al, 1993). En una inspección futura en $T_{i, k}$ pueden tenerse los siguientes posibles resultados: 1) no se detecta grieta por fatiga; 2) se detecta una grieta por fatiga, pero no se repara y 3 ) se detecta y repara una grieta por fatiga.

En el intervalo $0 \leq t \leq T_{l, k}$ la probabilidad de falla por fatiga de la junta $\mathrm{k}, k=1,2, \ldots, M$, se obtiene con la ecuación (7). En cualquier otro intervalo $T_{i-1, k} \leq t \leq T_{i, k}, i=1,2, \ldots, N_{k}$, la probabilidad total de falla por fatiga calculada tomando en cuenta todos los posibles resultados de las inspecciones pasadas y futuras es:

$$
p_{d, k}(t)=p_{d, k}\left(T_{i-1, k}\right)+\sum_{q=1}^{Q} P\left(E_{q} \cap M_{f, k}^{U}(t) \leq 0\right)
$$

donde $E_{q}$ representa una posible secuencia de resultados de inspecciones y/o reparaciones hasta $T_{i-1, k}$ , $Q$ es el número de todas las posibles secuencias de resultados hasta $T_{i-1, k}$. El índice $U$ indica que la probabilidad de falla de la junta $k$ depende de posibles cambios en el sistema producto de las posibles reparaciones y que la confiabilidad se actualiza con base en los resultados de las inspecciones previas.

El procedimiento para determinar el plan de inspección óptimo es el siguiente:

1. Se calculan los tiempos de inspección $T_{i, k}$ determinando el instante en que el índice de confiabilidad de una junta $k$ disminuye hasta alcanzar un nivel de confiabilidad requerido $\beta_{q}$. El índice de confiabilidad se actualiza en forma bayesiana (Moan et al, 1993) en cada tiempo de inspección $T_{i, k}$ suponiendo que no se detectan grietas por fatiga.

2. Se calcula la probabilidad total de daño por fatiga $p_{d, k}$ (ecuación 21) considerando todos los posibles resultados de una inspección en cada $T_{i, k}$ calculado (Moan et al, 1993). Se calculan las probabilidades anuales de falla (ecuación 2), la probabilidad acumulada de falla (ecuación 1) y los costos totales esperados asociados al $\beta_{q}$ requerido (ecuaciones 14, 15, 16 y 20). 
3. Los pasos 1) y 2) se repiten para diferentes valores de $\beta_{q}$ y se generan los planes de inspección para cada junta $k$. Así se tendrá un conjunto de costos totales esperados asociados a distintos niveles de confiabilidad requeridos $\beta_{q}$. Obsérvese que para el cálculo de los costos esperados de acuerdo con las ecuaciones 15,16 y 20 , se requiere haber evaluado la probabilidad anual de falla y la probabilidad acumulada de falla (ecuación 1)

4. Finalmente se selecciona como óptimo el plan de inspección asociado al valor de $\beta_{q}$ que tiene los costos esperados totales mínimos.

Los $\beta_{q}$ considerados deben ser mayores que un valor mínimo aceptable, el cual puede determinarse de las siguientes tres maneras (Flint, 1976; CIRIA, 1977; Faulkner, 1983; Jordaan, 1988; ISSC, 1991; Iwan et al., 1993; Paté-Cornell, 1993; Bhattacharya, et al, 2001):

1. A partir de la seguridad o nivel de riesgo implícito en códigos existentes, o a partir de la evaluación de estructuras existentes que se consideren aceptables.

2. A partir de fatalidades, daños ambientales o pérdidas de propiedades obtenidas con datos de accidentes ocurridos.

3. A partir de un análisis costo-beneficio, en el cual se consideren todos los peligros (e.g. cargas) y modos de falla (colapso, fatiga, etc.) así como las diferentes etapas de la vida de un sistema estructural (en operación en sitio y fases temporales asociadas con la fabricación, instalación y reparación) para cada una de las consecuencias de falla relevantes (fatalidades, contaminación, pérdida de bienes).

Siguiendo el procedimiento descrito, se elaboró el plan de inspección óptimo para la plataforma del caso de estudio. Se consideró un índice de confiabilidad mínimo aceptable para las juntas igual a 3.0, es decir una probabilidad de falla máxima aceptable igual a $1.3 \times 10^{-3}$. Se supuso un volumen diario de producción de la plataforma que la clasifica en la categoría de riesgo de muy altas consecuencias de falla de la norma NRF-003-PEMEX-2000 (PEMEX, 2000). De acuerdo con la norma, $R S R_{a d m}=1.9$ y la probabilidad anual de falla máxima aceptable a nivel global es $3.36 \times 10^{-4}$, la cual corresponde a un índice de confiabilidad de 3.4. Ésta es una probabilidad total de falla que incluye diferentes peligros, daños y mecanismos de falla. Aquí se considera que la probabilidad de falla aceptable por fatiga a nivel global es $10 \%$ de esta probabilidad total de falla (Straub y Faber, 2003), es decir $3.36 \times 10^{-5}$. Obsérvese de los resultados en la tabla 2 que la suma de las probabilidades de falla condicionales al daño por fatiga en las tres juntas es $4.2 \times 10^{-5}$. Dado que el cociente $3.36 \times 10^{-5} / 4.2 \times 10^{-5}$ es mayor que $1.3 \times 10^{-3}$, el valor de 3.0 adoptado para el índice de confiabilidad mínimo aceptable de las juntas satisface el requerimiento de confiabilidad global de la plataforma establecido en la norma. Por lo tanto, para determinar el plan de inspección óptimo se generaron planes de inspección para índices $\beta_{q}=3.00,3.15,3.30,3.45$ y 3.60 .

En la figura 8 se muestra el plan de inspección de la junta $\mathrm{N}^{\circ} 1$ para $\beta_{q}=3.00$, calculado con el módulo PROFAST del programa SESAM (Profast, 2002). La línea horizontal muestra el índice de confiabilidad aceptable $\beta_{q}=3.00$. Cabe mencionar que la junta no había sido inspeccionada hasta el momento y que por lo tanto no se tiene ningún tipo de información que permita realizar una actualización bayesiana de su confiabilidad. En la figura 8 se observa que el índice de confiabilidad de la junta es menor que el mínimo requerido $\beta_{q}=3.00$ lo cual obliga a marcar una inspección inmediata en el año presente 2006. Para planear la segunda inspección, el índice de confiabilidad se actualiza mediante la técnica bayesiana (Moan et al, 1993) suponiendo que en la primera inspección no se detecta grieta por fatiga. Los siguientes tiempos de inspección de la junta se obtienen con el mismo procedimiento. En la tabla 3 se muestran las fechas de inspección de las tres juntas críticas para los distintos valores de $\beta_{q}=3.00,3.15,3.30,3.45$ y 3.60. Una vez establecidos los años de inspección de cada junta, se calcula la probabilidad total de daño por fatiga considerando todos los posibles resultados de inspección en cada $T_{i, k}$. En la figura 9 se muestra la probabilidad total de 
daño por fatiga de la junta $\mathrm{N}^{\circ} 1$ para el caso en que $\beta_{q}=3.15$. La junta no ha sido inspeccionada desde la instalación de la plataforma hasta el año 2006 y por lo tanto no se cuenta con información que permita realizar una actualización bayesiana de su confiabilidad. La variación de la confiabilidad de la junta que se muestra en la figura 9 a partir del año 2006 está basada en los posibles resultados de las inspecciones planeadas usando la ecuación (21).

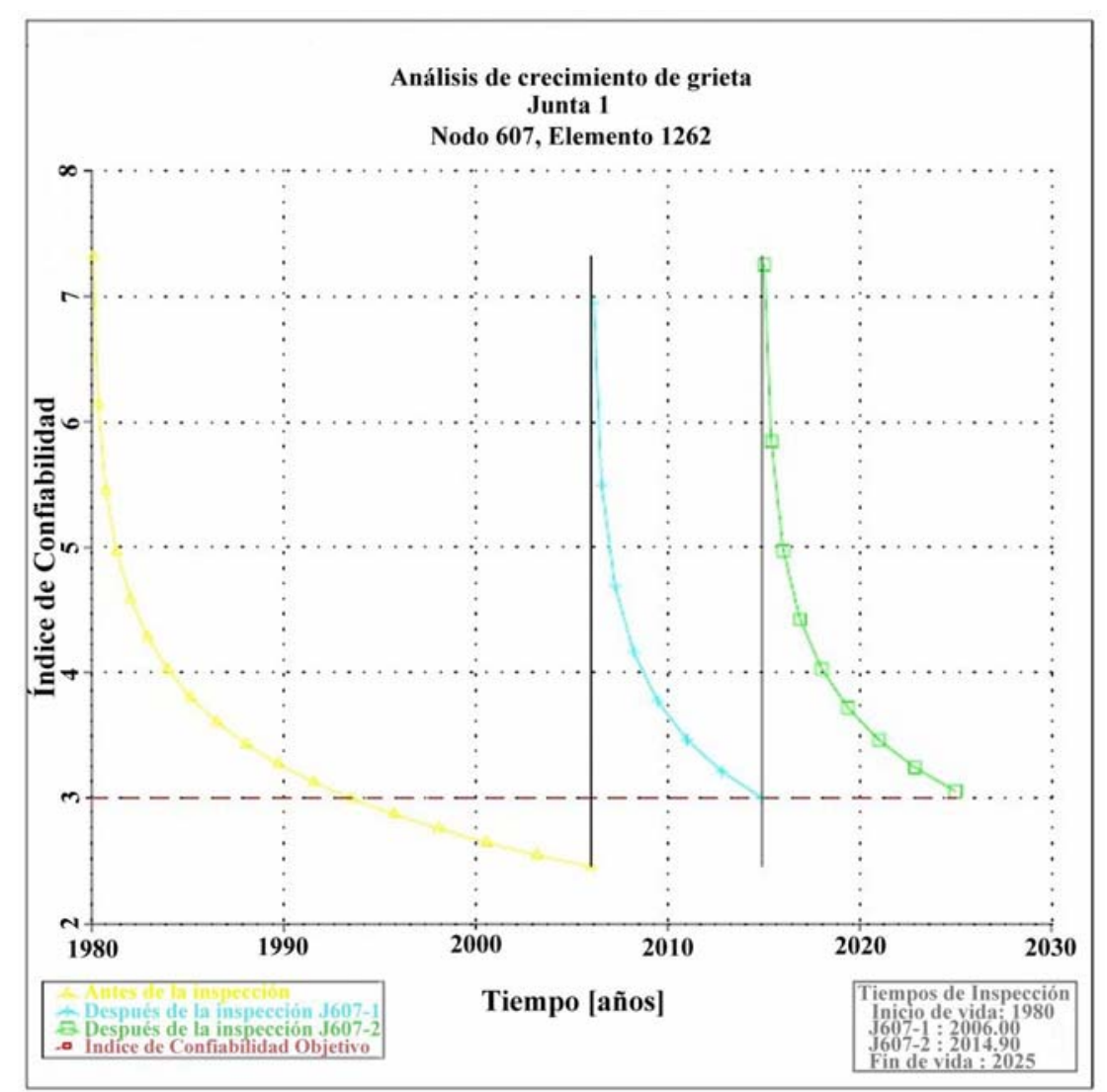

Figura 8. Evolución del índice de confiabilidad de la junta $\mathrm{N}^{\circ} 1$ y sus tiempos de inspección para $\beta_{q}=3.00$

Se observa que la confiabilidad siempre decrece debido: 1) al crecimiento de la grieta por el oleaje de operación y 2) a que los eventos que podrían incrementar la confiabilidad como el no detectar grieta o el de efectuar una reparación son aún inciertos. En la figura 10 se muestran los costos esperados de inspección y reparación asociados a los cinco planes de inspección mostrados en la tabla 3. Se consideró una tasa neta de descuento de $10 \%$. Como se nota en la figura 10 , el costo esperado de inspección crece con $\beta_{q}$. De acuerdo con la ecuación (15), esto sucede porque el número de inspecciones requeridas aumenta con $\beta_{q}$ y también porque la probabilidad acumulada de falla de la plataforma disminuye. En la figura 10 se observa también que los costos esperados de reparación son muy bajos y mucho menores que los costos esperados de inspección. La resistencia mínima requerida para reparación es igual a $R_{u, m i n}=R S R_{r e q} \times S_{r}=1.9 \times 431=818.9$ t. Obsérvese que la resistencia de la plataforma en el estado de daño más crítico es del orden de 1887 t, la cual es considerablemente mayor que la resistencia mínima requerida de 818.9t. Por lo tanto, según la ecuación (18), la probabilidad de reparación es muy pequeña lo que provoca que los costos esperados de reparación, dados por la ecuación (16), sean también muy pequeños. 
Tabla 3. Planes de inspección para diferentes valores de $\beta_{q}$

\begin{tabular}{ccl}
\hline Junta & $\beta_{\mathrm{q}}$ & \multicolumn{1}{c}{ Tiempos de inspección } \\
\hline & 3.00 & $2006.00-2014.90$ \\
1 & 3.15 & $2006.00-2013.29-2022.32$ \\
& 3.30 & $2006.00-2012.10-2019.67$ \\
& 3.45 & $2006.00-2011.08-2017.46-2024.62$ \\
& 3.60 & $2006.00-2010.23-2015.64-2021.71$ \\
\hline & 3.00 & $2006.00-2012.79-2021.15$ \\
2 & 3.15 & $2006.00-2011.72-2018.79$ \\
& 3.30 & $2006.00-2010.83-2016.83-2023.57$ \\
& 3.45 & $2006.00-2010.07-2015.20-2020.96$ \\
& 3.60 & $2006.00-2009.48-2013.87-2018.78-2024.12$ \\
\hline & 3.00 & $2006.00-2014.57$ \\
& 3.15 & $2006.00-2013.02-2021.72$ \\
& 3.30 & $2006.00-2011.75-2018.99$ \\
& 3.45 & $2006.00-2010.75-2016.81-2023.67$ \\
& 3.60 & $2006.00-2009.88-2014.99-2020.75$ \\
\hline
\end{tabular}

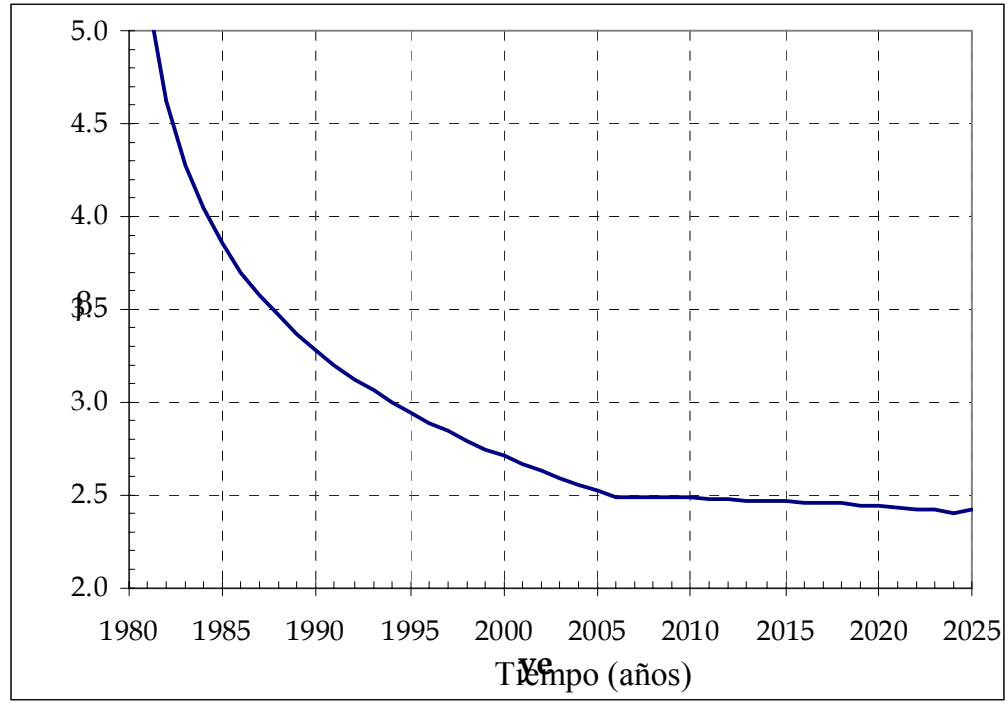

Figura 9. Índice de confiabilidad de la junta $\mathrm{N}^{\circ} 1$ considerando los 8 posibles eventos que resultan de las tres inspecciones futuras para $\beta_{q}=3.15$. 
Los costos esperados de falla y costos esperados totales se muestran en la figura 11. Se nota en la ecuación (20) que los costos futuros de falla deben disminuir con $\beta_{q}$. En la figura 11 se observa que esta disminución es poco importante. En esta aplicación los estados de daño no afectan significativamente la resistencia última de la plataforma (véase tabla 1) y, por lo tanto las probabilidades condicionales de falla para la condición intacta y para los estados de daño son muy cercanas entre sí (tabla 2). Esta condición genera, en la ecuación (2), que la probabilidad anual de falla $p_{a}(t)$ no cambie significativamente con $\beta_{q}$, ya que $p_{a, i} \approx p_{a, 0} ; i=1,2,3$ y por lo tanto $\beta_{q}$ no modificará de manera importante los costos futuros de falla.

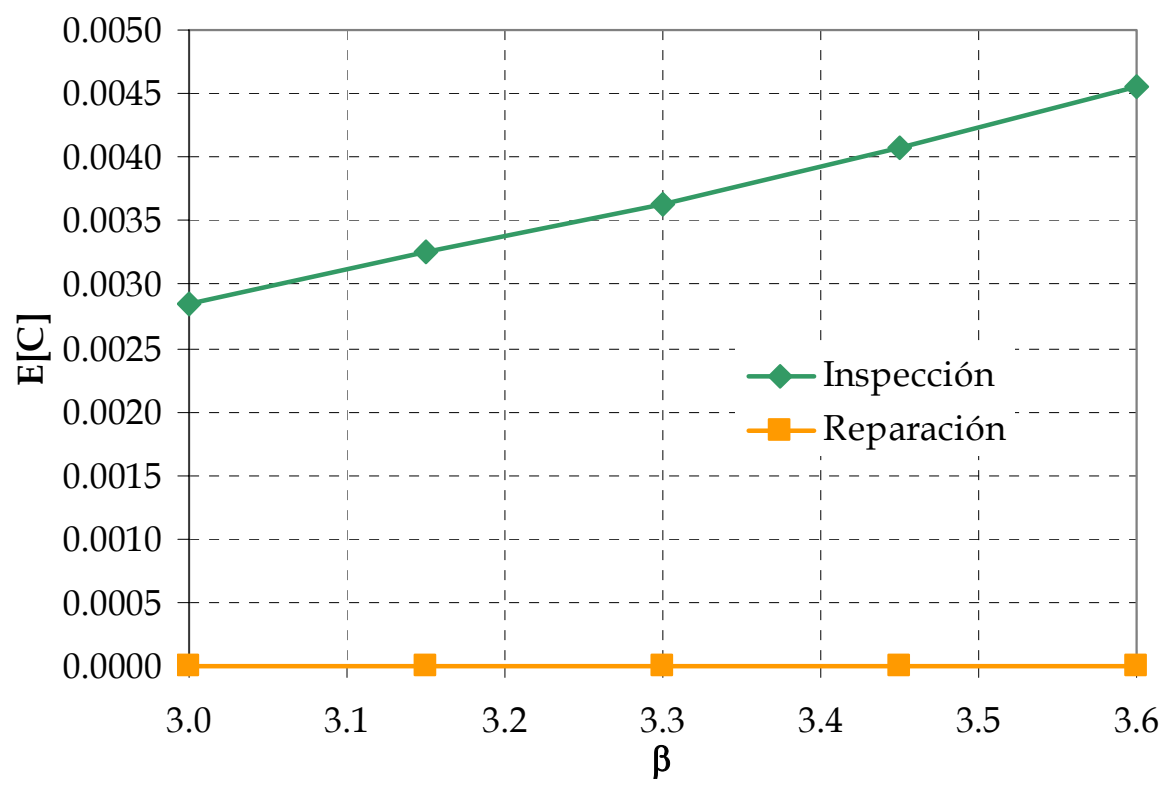

Figura 10. Costos esperados de inspección y reparación

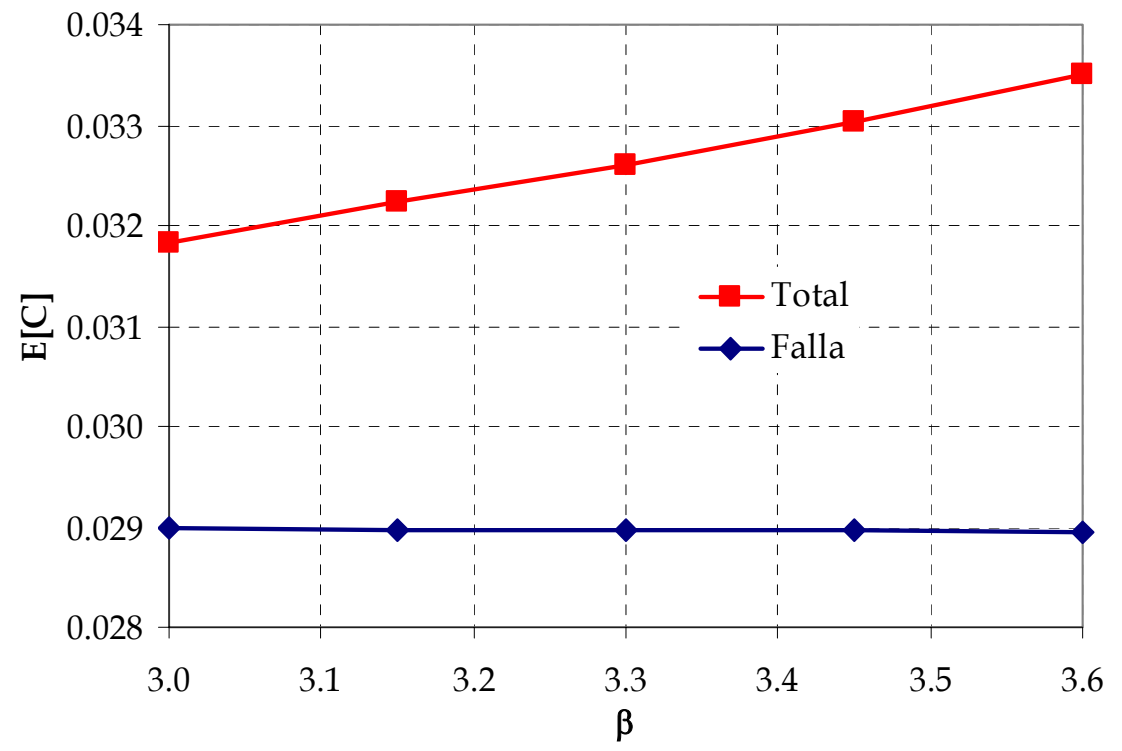

Figura 11. Costos esperados de falla y costos esperados totales. 
Así, debido a que: 1) los costos esperados de reparación son despreciables comparados con los costos esperados de inspección; 2) los costos esperados futuros de falla disminuyen ligeramente con $\beta_{q}$; y 3) sólo los costos esperados de inspección aumentan con $\beta_{q}$, la curva de costo total esperado mostrada en la figura 11 sigue la misma tendencia que la de los costos esperados de inspección. Se tiene entonces que el plan de inspección óptimo que minimiza el costo total esperado es el asociado a $\beta_{q}=3.00$. El plan de inspección óptimo se muestra en la tabla 4.

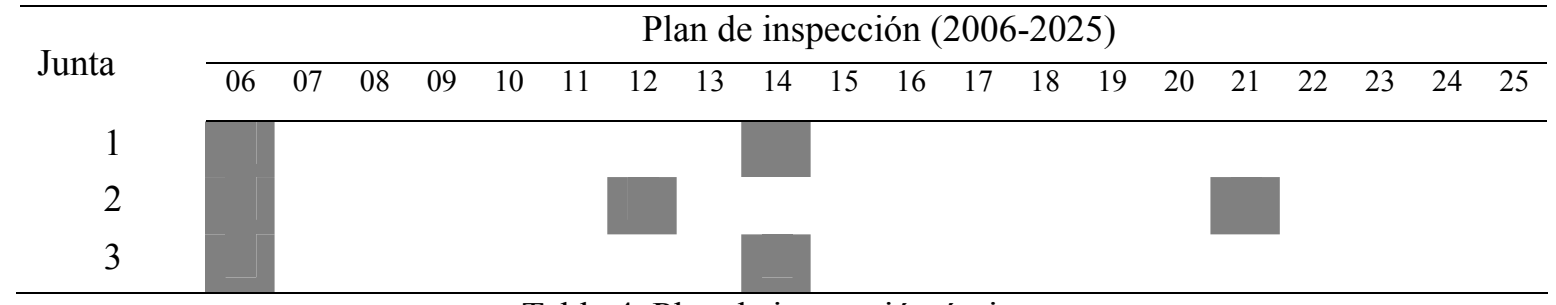

Tabla 4. Plan de inspección óptimo

\section{CONCLUSIONES}

En este trabajo se propuso un método para estimar la probabilidad de falla de plataformas marinas considerando la presencia de daño por fatiga en las juntas del jacket y sometidas a la acción de sismos. Para calcular la probabilidad condicional de falla dado un estado de daño por fatiga, se propuso una función de estado límite en términos del cortante basal sísmico actuante y del cortante basal resistente de la plataforma marina. Tanto la resistencia de la plataforma como el cortante sísmico basal actuante se modelaron como variables aleatorias lognormales. Se introdujeron sesgos tanto para la carga actuante como para la resistencia. Se propuso usar además un factor de carga para modificar las cargas sísmicas pseudoestáticas derivadas de un análisis de empuje lateral y así tomar en cuenta las características de ductilidad y resistencia residual de la plataforma.

El método propuesto se aplicó a un modelo estructural real, representativo de las instalaciones marinas existentes en México, y se evaluaron probabilidades de falla mediante simulación de Monte Carlo. Por otro lado, se planteó el procedimiento para calcular la probabilidad de falla por fatiga de las juntas del jacket con base en un modelo de mecánica de fractura elástica. Se estableció un modelo de riesgo para seleccionar el plan de inspección óptimo, definido como aquel que, satisfaciendo requerimientos mínimos de seguridad, optimiza los costos esperados. Se calcularon costos esperados de inspección, reparación y de falla, y se ilustró su aplicación en la elaboración del plan de inspección para extender la vida de servicio de una plataforma.

El trabajo presentado muestra el procedimiento completo para la generación de planes de inspección de plataformas marinas sometidas a daño por fatiga y expuestas a la acción de sismos. Con base en el acopio de información que resulte de análisis de empuje lateral de plataformas marinas, se podrá caracterizar de mejor manera las incertidumbres sobre la ductilidad y el factor de resistencia residual que, como se mostró, pueden influir de manera significativa en la evaluación de la probabilidad de falla de plataformas marinas. El efecto de otros tipos de daño y su incorporación en el análisis de riesgo para planeación de inspecciones será objeto de estudios futuros. El método propuesto se puede aplicar a otro tipo de estructuras sometidas a daño por fatiga. La aplicabilidad del método depende de que se cuente con modelos y herramientas de análisis que permitan estimar la probabilidad de falla de la estructura condicional a los estados críticos de daño por fatiga para los estados límite más relevantes. La determinación del plan de inspección óptimo, tal como se ha 
formulado en este artículo, requerirá adicionalmente de los índices de confiabilidad aceptables para cada estado límite.

\section{AGRADECIMIENTOS}

Los autores agradecen la colaboración de la M. en I. Claudia Rendón Conde del IMP en los análisis de resistencia última del modelo estructural. Se agradece también al Instituto Mexicano del Petróleo el apoyo a la estancia posdoctoral del primer autor en la cual se llevó a cabo la investigación cuyos resultados se presentan en este artículo.

\section{REFERENCIAS}

API RP-2A (1993), Recommended practice for planning, designing and constructing fixed offshore platforms, American Petroleum Institute, $20^{\text {a }}$ edición.

Bea, R (1992), "Seismic design and requalification methodologies for offshore platforms", Proceedings of the International Workshop on Seismic Design and Reassessment of Offshore Structures, California Institute of Technology.

Bea, R (1997), "Risk based oceanographic and earthquake criteria for design and requalification of platforms in the Bay of Campeche", Reporte a Petróleos Mexicanos, Instituto Mexicano del Petróleo y Brown and Root International Inc., Ocean Engineering Services, Moraga California.

Bhattacharya, B, R Basu y K Ma (2001), "Developing target reliability for novel structures: the case of the Mobile Offshore Base", Marine Structures, Vol. 14, pp.37-58.

Chávez, M (1997), "Seismic hazard and design spectra for Sourthern Gulf of Mexico", 16th International Conference on Offshore Mechanics and Artic Engineering, Proceedings of Earthquake Criteria Workshop: Recent Developments in Seismic Hazard and Risk Assessments for Port, Harbor, and Offshore Structures, Yokohama, Japón, Abril 17, ASME, New York, N.Y.

CIRIA (1977). "Rationalisation of safety and serviceability factors in structural codes". Reporte 63, London: CIRIA (Construction Industry Research and Information Association).

Enevoldsen I y J D Sørensen (1994), "Reliability-based optimization in structural engineering", Structural Safety, Vo.15, pp. 169-96.

Faber M H, S Engelund, J D Sørensen y A Bloch (2000). "Simplified and generic risk based inspection planning". Proceedings of the 19th Offshore Mechanics and Arctic Engineering Conference, New Orleans.

Faulkner, D (1983). "On selecting a target reliability for deep water tension leg platforms". Proceedings of the 11th IFIP Conference on System Modelling and Optimization. Copenhagen: IFIP.

Flint, A K (1976). "Design objectives for offshore structures in relation to social criteria". Proceedings of the BOSS Conference Trondheim: Tapir.

Heredia, E y R Montes (2004). "A bayesian model for the probability distribution of fatigue damage in tubular joints", Journal of Offshore Mechanics and Arctic Engineering, Journal of Offshore Mechanic and Artic Engineering, ASME, Vol. 126, pp. 243-249. 
Heredia, E, F L Silva, y R Montes (2006) "Reliability analysis of marine platforms subject to fatigue damage for risk based inspection planning", enviado para posible publicación al Journal of Offshore Mechanics and Arctic Engineering, OMAE-06-1068

ISSC (1988-94). Report of ISSC Committee IV.1. Design Philosophy. Proceedings of the 10th ISSC, (1988), 11th ISSC, Wuxi (1991) and 12th ISSC, St. John's, Newfoundland (1994).

Iwan, W D, C C Thiel, G W Housner y C A Cornell (1993). "A reliability-based approach to seismic reassessment of offshore platforms". Proceedings of the 6th ICOSSAR, Rotterdam: Balkema.

Jordaan, I (1988). "Safety levels implied in offshore structural codes: application to CSA program for offshore structures". Report, Memorial University of Newfoundland, St. John's. Prepared for the USA Program for Fixed Offshore Structures.

Kirkemo, F (1988), "Applications of probabilistic fracture mechanics to offshore structures", Applied Mechanic Review, Vol. 41, No 2, pp. 61-84.

Madsen H O, S Krenk y N Lind (1986), “Methods of Structural Safety”, Prentice-Hall Inc.

Madsen H O, J D Sørensen y R Olesen (1989). "Optimal inspection planning for fatigue damage of offshore structures". Proceedings of the 5th ICOSSAR, Vol. 3, pp. 2099-106.

Moan T, G O Hovde y A M Blanker AM (1993). "Reliability-based fatigue design criteria for offshore structures considering the effect of inspection and repair", Proceedings of 25th Annual Offshore Technology Conference.

Paris, P y F Erdogan (1963), "A critical analysis of crack propagation laws", ASME, Journal of Basic Engineering, Vol. 85, pp. 258-534.

Paté-Cornell, M E (1993). "Risk management for existing energy facilities: a global approach to numerical safety goals, in ageing of energy production and distribution systems". Applied Mechanics Reviews, Vol. 46, No. 5, New York: ASME.

PEMEX (2000), "Diseño y evaluación de plataformas marinas fijas en la Sonda de Campeche", NRF-003-PEMEX-2000, México.

PROFAST (2002). "Profast Theory, Probabilistic Fatigue Analysis". DNV Software Report No. 95-7005.

Silva, F L y E Heredia (2004), "Effect of uncertainties on the reliability of fatigue damaged systems", Proceedings of OMAE 2004, 23rd International Conference on Offshore Mechanics and Artic Engineering, OMAE2004-51485.

Skjong R (1985). "Reliability based optimization of inspection strategies". Proceedings of the ICOSSAR 85, Kobe, Japan, Vol. III, pp. 614-18.

Straub D y M Faber (2003), "Risk based acceptance criteria for joints subject to fatigue deterioration", Proceedings of the 22nd International Conference on Offshore Mechanics and Arctic Engineering, Paper No. OMAE2003-37224, México. 